\title{
Practical implementation of scheduling observations of moving objects that appear at random times
}

\author{
Nikolay Boldyrikhin ${ }^{1, *}$, Alexey Svizhenko ${ }^{1}$, Ivan Sosnovsky ${ }^{1}$, Anastasiya Manakova ${ }^{1}$ \\ ${ }^{1}$ Don state technical University, Rostov-on-Don, Russia
}

\begin{abstract}
The article is devoted to practical implementation of planning of observations of the set of mobile objects appearing in the area of visibility of the measuring information system at random moments of time. A variant of the implementation of the suboptimal distribution of the measuring resources of the information system between the problems of trajectory detection and estimation is proposed. Application of the attitude proposed in the article allows to increase accuracy of measurement of paths of mobile objects up to 52\% in comparison with uniform tracking plan.
\end{abstract}

\section{Introduction}

An essential reserve for improving the quality of functioning of complex measuring information systems (MIS) is optimization of control of measuring processes [1-11]. Tasks of observation control in MIS are control of accuracy of algorithms of measurement information processing at tracking of one or group of mobile objects [1-4,7-11].

Existing approaches to solving the problem of synthesis of laws of control of measuring processes are based on optimization of characteristics of control of observations in the task of filtering under the assumption that the fact of existence of information process is established, i.e. the problem of detection has already been solved. This assumption is not always justified, since in practice observation objects (OO) appear in the area of visibility of the MIS at previously unknown times. In addition, there may be situations when random is not only the time of appearance of objects in the area of visibility of the MIS, but also their number [1-4,9-11].

Therefore, it seems relevant to consider the best observation strategies in terms of a stochastic approach to models of observed objects appearing in an MIS visibility area, taking into account the multiplicity and random nature of $\mathrm{OO}$.

\section{Mathematical models of information and measurement processes in information systems}

The sequence of random events related to the appearance of $\mathrm{OO}$ in the area of visibility of

\footnotetext{
*Corresponding author: boldyrikhin@mail.ru
} 
MIS can be characterized by random moments of time of their appearance $t_{1}<t_{2}<\ldots$ on the time axis $t$. Such a sequence of events is referred to as a random flow or a point random process [12].

In general, any one-dimensional random stream may act as an OO pattern. The selection of the model describing the patterns of OO appearance in the area of the MIS visibility is carried out in accordance with the conditions of the specific task.

This approach can also be used effectively to plan observations of a single object. At the same time, different types of distributions can be used as a model of appearance of $\mathrm{OO}$ in the area of visibility of MIS depending on a specific practical task and volume of a priori information on the time of appearance of the object.

In most practical problems, it is assumed that the $\mathrm{OO}$ state vector is described by stochastic differential equation [7]

$$
\frac{d y(t)}{d t}=\phi(y(t), t)+g(y(t), t) \xi(t), y\left(t_{H}\right)=y_{H}, t \in\left[t_{H}, t_{\kappa}\right],
$$

$y(t) \in R^{n}$ - is the state vector; $y_{t}$ - is the random vector with density of distribution $W_{y_{u}}(y) ; t_{\mu}$ - is the moment of OO appearance in the area of MIS visibility; ${ }^{t_{\kappa}}$ - is the moment termination of observation; $g(y(t), t) \in R^{n \times r} ; \phi(y(t), t) \in R^{n} ; \phi, g$ - are nonlinear matrix functions measurable by a set of arguments satisfying the Lipschitz condition

$$
\mid \phi(y, t))\left.\right|^{2}+|g(y, t)|^{2} \leq M\left(1+|y|^{2}\right), M=\text { const } \geq 0,\left|g\left(y_{1}, t\right)-g\left(y_{2}, t\right)\right| \leq M\left|y_{1}-y_{2}\right|
$$

or any $y_{1}, y_{2} \in R^{n} ; \xi(t) \in R^{r} \quad$ - coloured noise, for which $M[\xi(t)]=0$; $M\left[\xi(t) \xi^{T}(t-\varsigma)\right]=Q_{\xi} \delta(\varsigma), Q_{\xi} \in R^{r \times r}$.

Expression (1) is a model of a continuous Markovian process that in many cases most fully reflects the physical essence of the phenomena to be examined.

As a mathematical model of process observation (1), the equation of the form is considered

$$
V(t)=\gamma(t) h(y(t), t)+\eta(t), t \in\left[0, t_{\kappa}\right]
$$

$V(t) \in R^{m}$ - is the observation vector; $h(y(t), t) \in R^{m}$ - is the nonlinear vector - function; $\gamma(t)$ is the scalar function whose meaning will be refined below; $\eta(t) \in R^{m}$ is the colored noise for which $M[\eta(t)]=0 ; M\left[\eta(t) \eta^{T}(t-\varsigma)\right]=Q_{\eta} \delta(\varsigma), Q_{\eta} \in R^{m \times m}$ is the diagonal matrix.

To solve the problem of estimating the information process (1), based on observation (3), various nonlinear filtering algorithms have been developed, a special case of which are the Kalman - Bucy linear filtering algorithms. To obtain the possibility of their application in the case when the models of information and measurement processes are described by equations (1), (3), respectively, the principle of linearization with respect to some reference trajectory $x_{o}(t)$ is used, which can be calculated, for example, based on the solution of the 
forecasting problem. When using this approach, the distribution density $y_{u}$ in (1) is approximated by a Gaussian frequency distribution.

Suppose that the distribution of a random value $t^{t_{H}}$ is known, denoted as $W\left(t_{H}\right)$. Into the subspace $\left[0, t_{\kappa}\right]$ of the space $[0, \infty]$ of possible values of the random variable $t_{H}$ discretization interval

$$
\begin{gathered}
\left\{t_{H}{ }^{0}, t_{H}{ }^{1}, \ldots, t_{H}{ }^{\mu}\right\}, \\
t_{H}{ }^{j+1}-t_{H}{ }^{j}=\Delta_{t}, j=\overline{0, \mu-1} .
\end{gathered}
$$

To obtain a reference trajectory, we form a set of solutions to an equation of the form

$$
\frac{d x_{0}\left(t_{H}{ }^{j}, t\right)}{d t}=\phi\left(x_{0}\left(t_{H}{ }^{j}, t\right), t\right), x_{0}\left(t_{H}{ }^{j}\right)=x_{0_{H}}, t \in\left[t_{H}{ }^{j}, t_{\kappa}\right] \text {. }
$$

Then the reference trajectory can be calculated using the approximate formula

$$
x_{o}(t)=\sum_{j=0}^{\mu-1} x_{o}\left(t_{H}{ }^{j}, t\right) w^{j}, w^{j}=W\left(t_{H}{ }^{j}\right) \Delta_{t} .
$$

We represent the state vector ${ }^{y}$ in the form

$$
y(t) \approx x_{0}(t)+x(t), x_{o}(t) \in R^{n}, x(t) \in R^{n} .
$$

Then, assuming the deviation vector $x(t)$ to be small, we obtain

$$
\begin{gathered}
\frac{d x(t)}{d t}=A(t) x(t)+F(t) \xi(t), t \in\left[t_{H}, t_{\kappa}\right], x\left(t_{H}\right)=x_{H}, \\
A(t)=\left[\left.\frac{\partial}{\partial x}\left(\phi(x(t), t)^{T}\right]^{T}\right|_{x=x_{o}(t)}, A(t) \in R^{n \times n}, F(t)=g\left(x_{o}(t), t\right), F(t) \in R^{n \times r}, x_{H}-\right. \\
\text { Gaussian vector, } M\left[x_{H}\right]=x_{H}^{*}, M\left[\left(x_{H}-x_{H}^{*}\right)\left(x_{H}-x_{H}^{*}\right)^{T}\right]=K_{H} .
\end{gathered}
$$

The observation equation (3) for the process (9) can be transformed to the form

$$
\rho(t)=\gamma(t) H(t) x(t)+\eta(t), t \in\left[0, t_{k}\right],
$$

$\rho(t) \in R^{m}$ - observation vector; $H(t)=\left[\left.\frac{\partial}{\partial x}\left(h(x(t), t)^{T}\right]^{T}\right|_{x=x_{o}(t)} ; H(t) \in R^{m \times n} ; \gamma(t)\right.$ scalar control function satisfying the following constraints

$$
\gamma(t) \in \Gamma=\{0,1\}, \int_{0}^{t_{\kappa}} \gamma(t) d t=t_{\Sigma}<t_{\kappa}
$$


If the composition of the measured parameters can be varied, i.e. the structure of the matrix $H(t)$ takes into account the constraint

$$
H(t) \in \bar{H}(t)
$$

where the set $\bar{H}(t)$ characterizes the potentially possible composition of the measuring instruments.

The possibility of planning the observation process consists in the ambiguity of assigning the functions $H(t)$ and $\gamma(t)$. At the same time, $\gamma(t)$ it describes the program of operation of the measuring instruments in time (measurement program). If $\gamma(t)=1$, then at the moment $\mathrm{t}$ a measurement is made, if $\gamma(t)=0$ - measurements are not made.

The structure of the set $\bar{H}(t)$, as well as the method of choosing admissible elements from it, are determined by a specific technical problem.

Models (9), (10) can be provided with a Kalman filter, for which the following expressions are valid:

$$
\begin{gathered}
\frac{d x^{*}(t)}{d t}=A(t) x^{*}(t)+\gamma(t) K(t) H^{T}(t) Q_{\eta}^{-1}\left[\rho(t)-H(t) x^{*}(t)\right], x^{*}\left(t_{H}\right)=x^{*}, \\
\frac{d K(t)}{d t}=A(t) K(t)+K(t) A(t)^{T}+C(t)-\gamma(t) K(t) B(t) K(t) \\
K\left(t_{H}\right)=K_{H}, t \in\left[t_{H}, t_{\kappa}\right],
\end{gathered}
$$

$x^{*}(t) \in R^{n}$ - estimate of the state vector; $K(t) \in R^{n \times n}$ - covariance matrix of estimation errors;

$$
C(t)=F(t) Q_{\xi} F(t)^{T} ; B(t)=H(t)^{T} Q_{\eta}^{-1} H(t)
$$

The aggregate

$$
\Pi=\{\gamma \in \Gamma, H \in \bar{H}\},
$$

forms an observation plan.

In the case of evaluating the set of information processes that appear in the visibility zone in accordance with the laws of a stochastic flow, equations (9), (10), (13) and (14) take the form respectively:

$$
\frac{d x_{i}}{d t}=A_{i} x_{i}+F_{i} \xi_{i}, \quad i=1,2, \ldots, \quad x_{i}\left(t_{\mu i}\right)=x_{i \mu}
$$

$x_{i}=x_{i}(t) \in R^{n_{i}}-$ state vector of the $i$-th object; $x_{i H}-$ Gaussian vector; $M\left[x_{i H}\right]=x_{i н}^{*}$, $M\left[\left(x_{i H}-x_{i H}^{*}\right)\left(x_{i H}-x_{i H}^{*}\right)^{T}\right]=K_{i H} ; t_{\mu i}$ - a random moment of the appearance of the $i$-th object, obeying the laws of the flow of random events; $A_{i}=A_{i}(t) \in R^{n_{i} \times n_{i}}$; $F_{i}=F_{i}(t) \in R^{n_{i} \times r_{i}} ; \xi_{i}=\xi_{i}(t) \in R^{r_{i}}-i-$ th colored noise for which; $M\left[\xi_{i}(t)\right]=0$; $M\left[\xi_{i}(t) \xi_{i}^{T}(t-\varsigma)\right]=Q_{i \xi} \delta(\varsigma), Q_{i \xi} \in R^{r_{i} \times r_{i}}$ 
$\rho=\sum_{i=1}^{I\left(t_{\mathrm{k}}\right)} f_{i} \gamma_{i} H_{i} x_{i}+\eta, \quad t \in\left[0, t_{\mathrm{K}}\right]$,
$\rho=\rho(t) \in R^{m} ; \quad f_{i}=f_{i}\left(t_{\mu i}, t\right)= \begin{cases}1, & t_{u i} \leq t \leq t_{\mathrm{K}}, \\ 0, & t<t_{u i}, \quad t_{u}>t_{\mathrm{K}} ; \quad \gamma_{i}=\gamma_{i}(t)-i \quad \text { th control }\end{cases}$ function; $H_{i}=H_{i}(t) \in R^{m \times n_{i}}$ - matrix function that determines the composition of the measured parameters of the $i-$ th object; $\eta=\eta(t) \in R^{m}-$ colored noise for which $M[\eta(t)]=0 \quad ; \quad M\left[\eta(t) \eta^{T}(t-\varsigma)\right]=Q_{\eta} \delta(\varsigma), Q_{\eta} \in R^{m \times m}-{ }_{\text {diagonal matrix; }} I(t)-$ integer discrete random process; $I\left(t_{\kappa}\right)-$ describes the number of OO from the set (16)

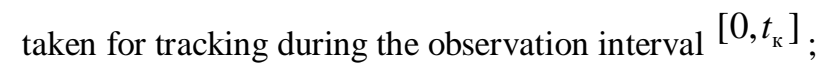

$$
\begin{gathered}
\frac{d x_{i}^{*}}{d t}=A_{i} x_{i}^{*}+\gamma_{i} K_{i} H_{i}^{T} Q_{\eta}^{-1}\left[\rho-H_{i} x_{i}^{*}\right], x^{*}\left(\tau_{i}\right)=x_{i t}^{*}, \\
\left.\frac{d K_{i}}{d t}=A_{i} K_{i}+K_{i} A_{i}^{T}+C_{i}-\gamma_{i} f_{i} K_{i} B_{i} K_{i}, i=\overline{1, I\left(t_{\mathrm{K}}\right.}\right), t \in\left[t_{\mu i}, t_{\mathrm{K}}\right] \\
K_{i}\left(t_{\mu i}\right)=K_{i t}, \tau_{i} \in\left[0, t_{\kappa}\right], \\
\text { where } C_{i}=F_{i} Q_{i \xi} F_{i}^{T} ; B_{i}=H_{i}^{T} Q_{\eta}^{-1} H_{i} .
\end{gathered}
$$

In this case, the restrictions on the control functions $\gamma_{i}(t)$ and matrices $H_{i}$ that determine the composition of the measured parameters will take the following form

$$
\gamma_{i}(t) \in \Gamma=\{0,1\}, \sum_{i=1}^{I\left(t_{k_{k}}\right)} \gamma_{i}(t) \in \Gamma \int_{0}^{t_{\kappa}} \sum_{i=1}^{I\left(t_{k}\right)} \gamma_{i}(t) d t=t_{\Sigma}<t_{\kappa}, H_{i}(t) \in \bar{H}_{i}(t) .
$$

Constraints (20) determine the range of problems to be solved, i.e. The calculations presented below correspond to the conditions of operation of the MIS with time division of channels: at the same moment of time it can track only one object, the rest of the trajectories at this time are calculated by extrapolation.

In this case, the set of

$$
\left\{\Pi_{i}, i=\overline{1, I\left(t_{k}\right)}\right\}=\Pi, \quad \Pi_{i}=\left\{\gamma_{i} \in \Gamma, H_{i} \in \overline{H_{i}}\right\}
$$

is called the plan of observation.

The statement of the problem of optimization of measuring processes as a problem of programmed control of a dynamic system (19) can be formalized based on the criterion of L-optimality:

$$
J=M\left[\sum_{i=1}^{I\left(t_{\mathrm{K}}\right)} \omega_{i}^{T} K_{i}\left(t_{\mathrm{\kappa}}\right) \omega_{i}\right] \rightarrow \min _{\Pi}
$$


Formula (22) characterizes the accuracy of the scalar process estimation $\sum_{i=1}^{I\left(t_{\mathrm{K}}\right)} \omega_{i}^{T} x_{i}(t)$ the moment of time $t=t_{\kappa}$.

The L-optimality criterion corresponds to the physical meaning of a large class of control problems for measurement processes.

Let us set the problem to determine ${ }^{\left[0, t_{\kappa}\right]}$ the optimal, in the sense of (22), strategy for observing (21) the flow of $\mathrm{OO}$, the trajectories of which are described by expression (16).

It should be noted that the use of the mathematical model (19) is rather cumbersome due to its high dimension and nonlinearity [7]. Using the approach proposed in [7], we pass to the projection of the Hamiltonian system corresponding to (19) onto the space $R^{2 n_{i}}$ of variables:

$$
\begin{gathered}
\frac{d q_{i}}{d t}=-A_{i}^{T} q_{i}+\gamma_{i} f_{i} B_{i} p_{i}, \\
\frac{d p_{i}}{d t}=C_{i} q_{i}+A_{i} p_{i}, \quad i=\overline{1, I\left(t_{\kappa}\right)}, \quad t \in\left[t_{\mu i}, t_{\kappa}\right], \\
p_{i}=p_{i}(t) \in R^{n_{i}}, \quad q_{i}=q_{i}(t) \in R^{n_{i}}, K_{i} q_{i}=p_{i}, \quad \forall \quad t, \quad t \in\left[t_{H i}, t_{\kappa}\right] .
\end{gathered}
$$

For the times when objects appear, the following relation is valid

$$
\begin{aligned}
& K_{i 0} q_{i 0}=p_{i 0}, \quad i=\overline{1, I\left(t_{\kappa}\right)}, \\
& q_{i 0}=q_{i}\left(t_{H i}\right), \quad p_{i 0}=p_{i}\left(t_{H i}\right), t_{H i} \in\left[0, t_{\kappa}\right] .
\end{aligned}
$$

Criterion (22), taking into account (23), (24), can be written in the form

$$
\begin{gathered}
\hat{J=} M\left[\sum_{i=1}^{I\left(t_{\kappa}\right)} \omega_{i}^{T} p_{i}\left(t_{\kappa}\right)\right] \rightarrow \min _{\Pi}, \\
q_{i}\left(t_{\kappa}\right)=\omega_{i} .
\end{gathered}
$$

The conditions of the optimization problem are described by a set of fictitious dynamical systems (23) and a quality criterion (25). Their feature is associated with the presence of a priori unknown times of the appearance of mobile air objects $t_{m i}$ and with a priori uncertainty regarding the number of OOs filtered on $\left[0, t_{\kappa}\right]$.

\section{Implementation of the planning procedure}

In [1-4, 9-11], the mathematical formulation of the problem considered above and the procedure for its solution are presented in detail. Observations are planned on the basis of the following relationships describing the program functions $T_{i}^{o n}(t)$ and the composition of the measured parameters $H_{i}^{o n}(t)$ : 
$\psi_{i}=\int_{0}^{t_{H}} W_{i}\left(t_{H}\right) d t_{u}, \quad \hat{W}_{i}\left(t_{H}\right)=W_{i}\left(t_{H}\right) / \psi_{i}, W_{i}\left(t_{H}\right)$ - distribution density of the moment of appearance of the i-th object in the visibility zone of the information system

$$
B_{i}(t)=\left(H_{i}(t)\right)^{T} Q_{h}^{-l}(t) H(t)_{i} .
$$

The decision rule for constructing an observation plan is described by the formula

$$
\gamma_{i}^{o n}(t)=\left\{\begin{array}{lll}
1, & T_{i}^{o n}(t) \geq \alpha, & T_{i}^{o n}(t) \geq T_{s}^{o n}, \quad\left(s=\overline{1, I\left(t_{k}\right)}, \quad s \neq i\right), \\
0, & T_{i}^{o n}(t)<\alpha, \quad T_{i}^{o n}(t)<T_{s}^{o n},
\end{array}\right.
$$

where the Lagrange $\alpha-$ multiplier associated with the constraint, $\int_{0}^{t_{\kappa}} \sum_{i=1}^{I\left(t_{\kappa}\right)} \gamma_{i}(t) d t=t_{\Sigma}<t_{\kappa}$, $(\alpha \neq 0)$.

Let's assume that as a result of implementing the above procedure, a plan of observations is synthesized (Fig. 1 a). This plan is a priori, and involves the observation of two objects in accordance with the specified probabilistic characteristics of the moments of their appearance in the visibility zone of the MIS. The probability of a third process occurring during the observation interval is so small that the program function associated with it does not affect the formation of the observation plan [4]. However, in real-time observation mode, a third and other objects may appear, i.e. the resulting a priori plan can only be used as a reference plan, which must be adjusted directly during observations.

Let's consider the procedure for adjusting the a priori plan directly in the course of observations (Fig. 1).

Let's assume that the 1 st object was detected at some point in time $t^{1} \in\left[0, t_{1}\right)$, then starting from this point in time, the resource of the information system is spent on tracking the 1st object and on detecting the next ones (Fig. $1 \mathrm{~b}$ ). If at some point in time $t^{2} \in\left[t_{0}, t_{1}\right)$ there was a detection of the 2nd object, then starting from this point in time, the resource of the information system is distributed between the tasks of evaluating the trajectories of the 1st and $2 \mathrm{nd} \mathrm{OO}$ and detecting the following processes. In this case, the time used for detection is determined by a certain minimum value $t_{\text {min } s}$, during which objects that are in the visibility zone are guaranteed to be detected. The time interval allocated for evaluating the trajectory of the $2 \mathrm{nd} \mathrm{OO}$ is also determined by the minimum value $t_{\operatorname{mino}}$ ("minimum" evaluation mode), the rest of the time is spent on evaluating the first process. This is due to the fact that according to the a priori plan (Fig. 1 a), the interval $\left[t_{0}, t_{1}\right)$ is defined as the interval of priority evaluation of the first information process. Let's call this mode "priority". 
If one or more objects are detected in the interval $\left[t_{0}, t_{1}\right)$, all of them will be evaluated in the "minimum" mode.

During the interval $\left[t_{1}, t_{\kappa}\right]$, the resource of the information system is also spent on detecting and tracking two objects, but now the second object is accompanied in "priority" mode (Fig. 1 b). If at some point $t^{2} \in\left[t_{1}, t_{\kappa}\right]$ in time there was a detection of the 3 rd object, the MIS resource is spent on tracking the 2nd OO in "priority" and the 3rd OO in the minimum mode (Fig. 1 in). If the second and third ones are not detected during the entire observation interval, the plan shown in figure 1d is used.

Example of solving the problem. Let's look at the solution of the problem using a simple example. When modeling the algorithm, the following restrictions were introduced.

$$
\begin{gathered}
n_{i}=1, \quad A_{i}(t) \equiv a_{i}(t), H_{i}(t) \equiv 1, \quad F_{i}(t) \equiv 1, \\
\omega_{i} \equiv \omega, Q_{i \xi} \equiv Q_{\xi}, K_{i 0} \equiv K_{0} .
\end{gathered}
$$

a)

$$
\gamma_{i}(t)
$$

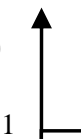

b)

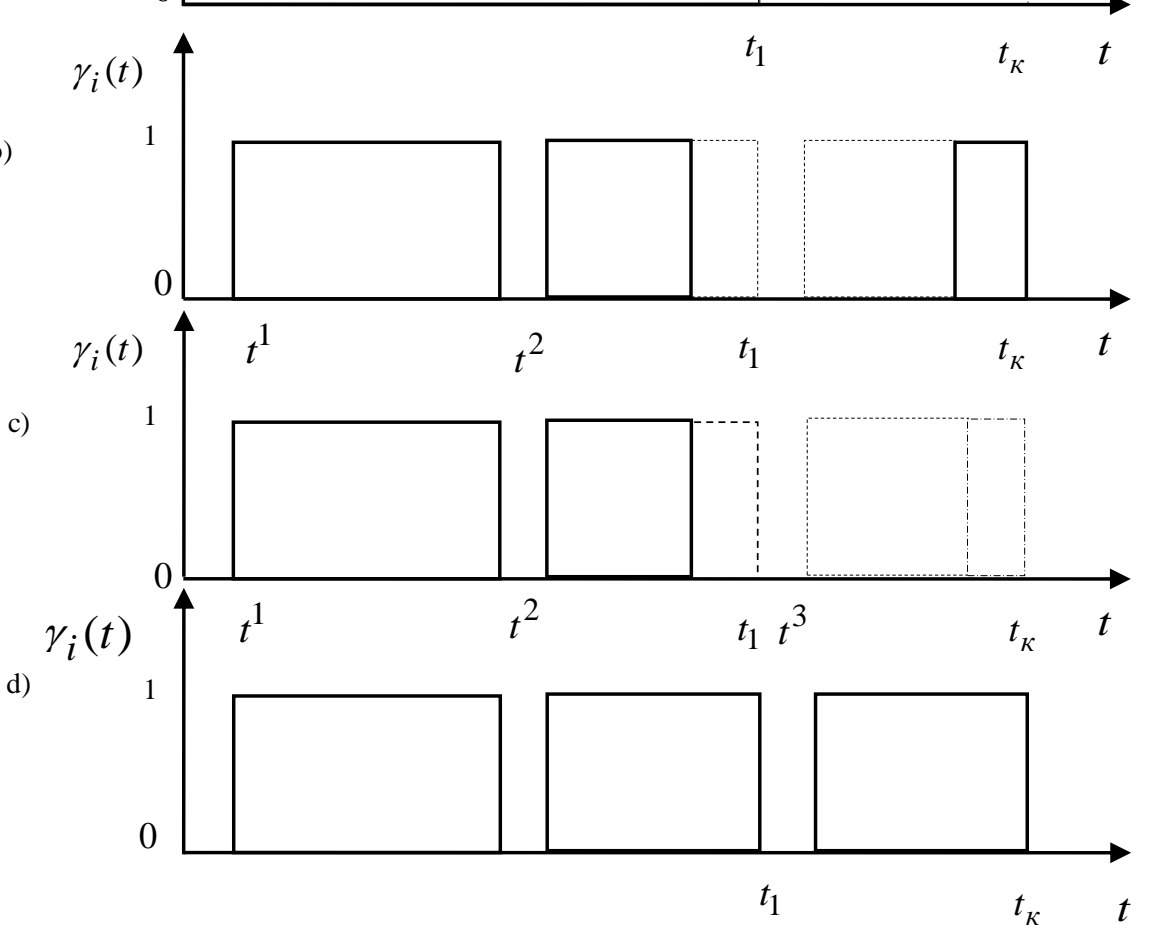

- $\gamma_{1}(t)$ - control function for the 1 st object

- $\quad \gamma_{2}(t)$ - control function for the 2 nd object

Fig. 1. Correction of the a priori observation plan. 
All the values given in the example were assumed to be dimensionless for simplicity. The simulation was performed with the following initial data:

$$
\begin{aligned}
& a_{1}(t)=0.68 / t, a_{2}(t)=0.6 / t, a_{3}(t)=0.58 / t, Q_{\xi}=0.5, K_{0}=0.01 \\
& \omega=0.1, Q_{\eta}=4, \mu=30, \Delta_{\tau}=1, t_{\kappa}=30, t_{\Sigma}=27, v=0.04, \bar{\psi}=0.1
\end{aligned}
$$

The initial plan of observations is shown in figure 2.

During the modeling process, it was found that $T_{3}^{1}(t)<\min \left\{T_{1}^{1}, T_{2}^{1}\right\}$, i.e., at the first iteration, the program function for the 3rd object did not affect the process of forming the observation plan. This pattern has been preserved in subsequent iterations.

The structure of program functions in the third iteration is shown in figure 3.

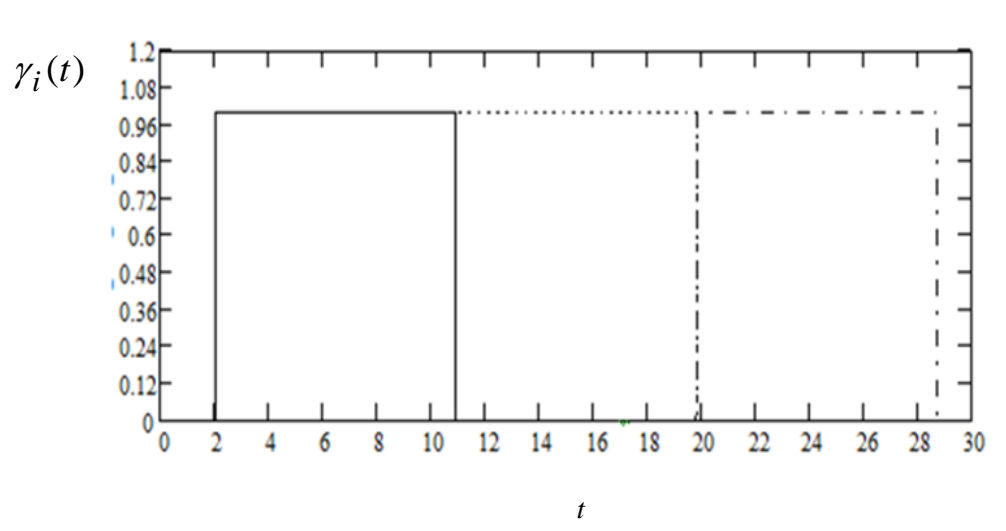

- $\gamma_{1}(t)$ - Control function for the 1 st object

- - $\gamma_{2}(t)$ - Control function for the 2 nd object

-. $\gamma_{3}(t)$ - Control function for the 3 rd object

Fig. 2. Initial plan of observations.

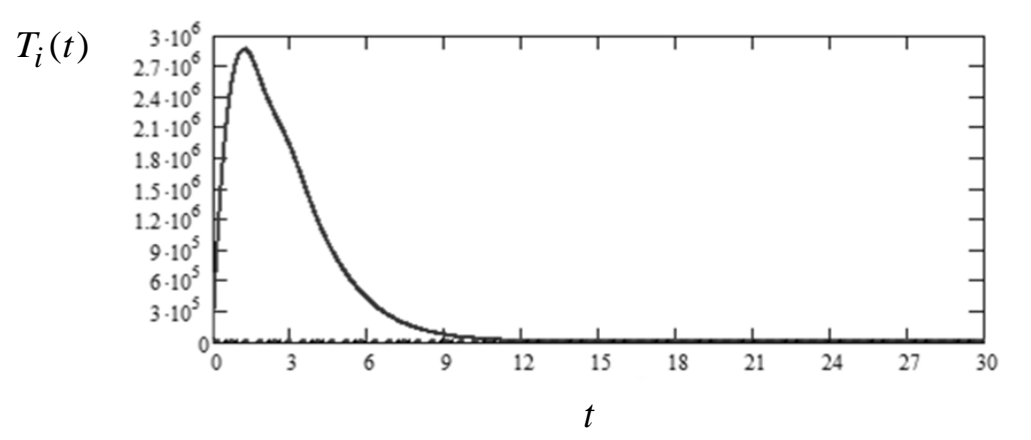

$-T_{1}(t)$ - program function for the 1 st object

- $-T_{2}(t)$ - program function for the 2 nd object

Fig. 3. Program functions in the third iteration. 
Starting from the 3rd interval of the iterative procedure, changes in program functions, and as a result, the laws of observation management, practically do not occur. Therefore, the observation plan corresponding to $k=3$ it can be considered suboptimal.

Figure 4 shows a suboptimal observation plan.

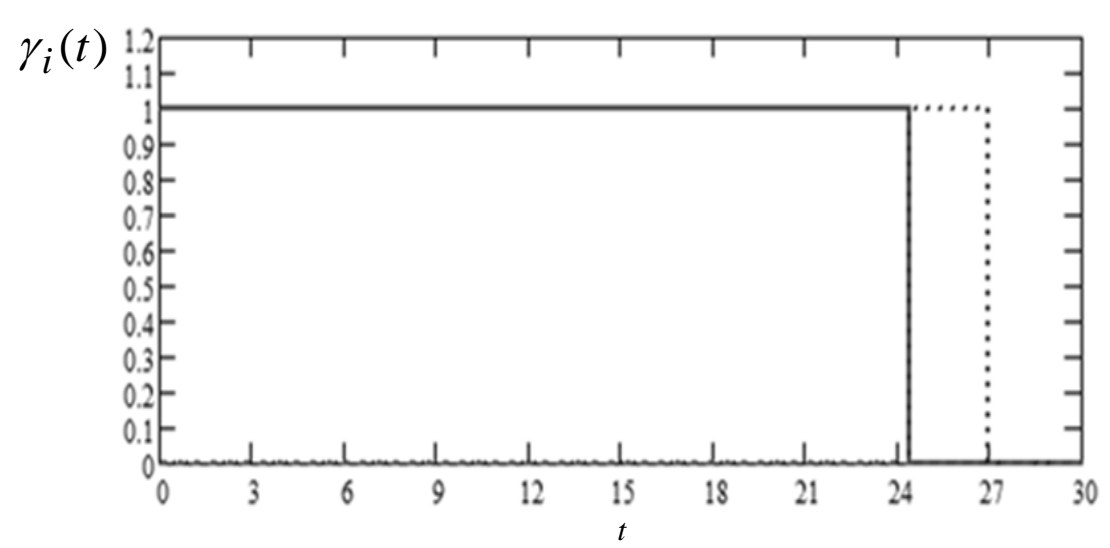

- $\gamma_{1}(t)$ - control function for the 1 st object

- $\gamma_{2}(t)$ - control function for the 2 nd object

Fig. 4. Suboptimal observation plan.

The gain exactly compared to the original plan was

$$
\delta=\frac{\mathrm{J}^{0}-\mathrm{J}^{3}}{\mathrm{~J}^{0}} \times 100 \%=52 \% \text {. }
$$

It should be noted that the resulting gain of $52 \%$ determines the upper potential limit. In a real situation, the observation plan may differ significantly from the a priori one, so the degree of approximation to the potential boundary depends on how significant these differences are. For example, figure 5 shows a plan of observations when the first object is detected at a time $t=3$. Observation of the second object in accordance with the a priori plan was assumed from the moment $t=24.2$, so a detection session was held in advance, during which the second object was detected at the moment $t=23$. The third object was not detected. The $\delta$ value was $43 \%$ compared to the uniform plan shown in figure 2. 


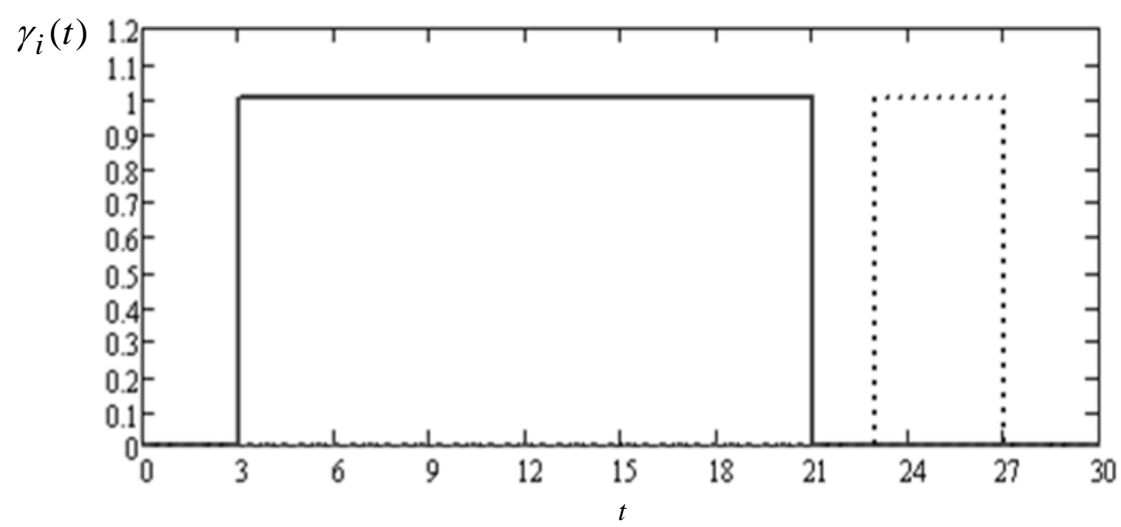

- $\gamma_{1}(t)$ - control function for the 1 st object

- - $\gamma_{2}(t)$ - control function for the 2 nd object

Fig. 5. Real observation plan.

Thus, the given example allowed us to illustrate the features of practical implementation of planning observations of mobile aerial objects that appear at random moments of time.

\section{References}

1. Boldyrikhin N V, Korneev A A, Goryushkin S V 2008 Bulletin of the Volgograd State Technical University 8(46) 11-14

2. Boldyrikhin N V, Safaryan, O A, Razumov P V, et al 2020 ICCAIS 2020 - 3rd International Conference on Computer Applications and Information

3. Boldirikhin N, Safaryan O, Svizhenko A 2019 Radiation and Scattering of Electromagnetic Waves (RSEMW) DOI: 101109/RSEMW20198792788

4. Boldyrikhin N V, Khutortsev V V 2006 Automation and Telemechanics 67(12) 19001912

5. Grigoriev F N, Kuznetsov N A, Serebrovsky A P 1986 Observation management in automatic systems (M: Nauka) p 218

6. Kondratyev V S, Kotov A F, Markov L N 1986 Multi-position radio engineering systems (M: Radio and communication) p 264

7. Malyshev V V, Krasil'shchikov M M, Karlov V I 1989 Optimization of observation and control of aircraft (M: Mashinostroenie) p 312

8. Tikhonov V I 1965 Statistical theory of radio engineering devices (M.: VVIA named after Professor Zhukovsky) p 420

9. Svizhenko A A, Khutortsev V V 2010 Journal of Computer and Systems Sciences International 49(3) 372-379

10. Khutortsev V V, Boldyrikhin N V 2005 Automatic Control and Computer Sciences 39(5) 9-19

11. Khutortsev V V 2013 Automatic Control and Computer Sciences 47(1) 48-56

12. Kingman J 2007 Poisson Processes ( M .: MTsNMO) p 136 